\title{
Scalar and pseudoscalar meson pole terms in the hadronic light-by-light contributions to $a_{\mu}^{h a d}$
}

\author{
E. Bartoš ${ }^{1}$, A.-Z. Dubničkovái ${ }^{1}$, S. Dubnička ${ }^{2}$, E. A. Kuraev ${ }^{3}$ \\ and E. Zemlyanaya ${ }^{4}$
}

1 Dept. of Theor. Physics, Comenius Univ., Bratislava, Slovak Republic

2 Inst. of Physics, Slovak Acad. of Sci., Bratislava, Slovak Republic

3 Bogoliubov Lab. of Theor. Physics, JINR Dubna, 141980 Dubna, Russia

${ }^{4}$ Lab. of Information Technologies, JINR Dubna, 141980 Dubna, Russia

\begin{abstract}
Third QED order hadronic light-by-light (LBL) contributions $a_{\mu}^{L B L}(M)$ to the anomalous magnetic moment of the muon $a_{\mu}^{\text {had }}$ from the pole terms of scalar $\sigma, a_{0}(980)$ and pseudoscalar $\pi^{0}, \eta, \eta^{\prime}$ mesons $(M)$ in the framework of the linearized extended Nambu-Jona-Lasinio model are evaluated. The off-shell structure of the photon-photonmeson vertices is taken into account by means of constituent quark triangle loops. The mass of the quark is taken to be $m_{u}=m_{d}=m_{q}=$ $(280 \pm 20) \mathrm{MeV}$. The unknown strong coupling constants of $\pi^{0}, \eta, \eta^{\prime}$ and $a_{0}$ mesons with quarks are evaluated in a comparison of the corresponding theoretical two-photon widths calculated in the framework of our approach with experimental ones. The $\sigma$-meson coupling constant is taken to be equal to $\pi_{0}$-meson coupling constant as it follows from the linearized Nambu-Jona-Lasinio model Lagrangian. Then one obtains $a_{\mu}^{L B L}\left(\pi_{0}\right)=(81.83 \pm 16.50) \times 10^{-11}, a_{\mu}^{L B L}(\eta)=(5.62 \pm 1.25) \times$ $10^{-11}, a_{\mu}^{L B L}\left(\eta^{\prime}\right)=(8.00 \pm 1.74) \times 10^{-11}, a_{\mu}^{L B L}(\sigma)=(11.67 \pm 2.38) \times 10^{-11}$ and $a_{\mu}^{L B L}\left(a_{0}\right)=(0.62 \pm 0.24) \times 10^{-11}$. The total contribution of meson poles in LBL is $a_{\mu}^{L B L}(M)=(107.74 \pm 16.81) \times 10^{-11}$.
\end{abstract}




\section{Introduction}

The muon is described by the Dirac equation and its magnetic moment is related to the spin by means of the expression

$$
\vec{\mu}=g\left(\frac{e}{2 m_{\mu}}\right) \vec{s}
$$

where the value of the gyromagnetic ratio $g$ is predicted (in the absence of the Pauli term) to be exactly 2 .

In fact, however, the interactions existing in nature modify $g$ to be exceeding the value 2 because of the emission and absorption of virtual photons (electromagnetic effects), intermediate vector and Higgs bosons (weak interaction effects) and the vacuum polarization into virtual hadronic states (strong interaction effects).

In order to describe this modification of $g$ theoretically, the magnetic anomaly was introduced by the relation

$$
\begin{aligned}
a_{\mu} \equiv & \frac{g-2}{2}= \\
& a_{\mu}^{(1)}\left(\frac{\alpha}{\pi}\right)+\left(a_{\mu}^{(2) Q E D}+a_{\mu}^{(2) h a d}\right)\left(\frac{\alpha}{\pi}\right)^{2}+a_{\mu}^{(2) w e a k}+O\left(\frac{\alpha}{\pi}\right)^{3}
\end{aligned}
$$

where $\alpha=1 / 137.03599976(50)$ [1] is the fine structure constant.

Here we would like to make a note that from all three charged leptons $\left(e^{-}, \mu^{-}, \tau^{-}\right)$the muon magnetic anomaly is the most interesting object for theoretical investigations due to the following reasons:

i) it is one of the best measured quantities in physics [2]

$$
a_{\mu}^{e x p}=(116592020 \pm 160) \times 10^{-11}
$$

ii) its accurate theoretical evaluation provides an extremely clean test of "Electroweak theory" and may give hints on possible deviations from Standard Model (SM)

iii) the new measurement [3] in BNL is expected to be performed with a definitive accuracy

$$
\Delta a_{\mu}^{e x p}= \pm 40 \times 10^{-11}
$$

i.e. it is aimed at obtaining a factor 4 in a precision above that of the last E-821 measurements (3). 
At the aimed level of the precision (四) a sensibility will already exist to contributions 4, 5]

$$
a_{\mu}^{(2,3) w e a k}=(152 \pm 4) \times 10^{-11},
$$

arising from single- and two-loop weak interaction diagrams.

However, as we have mentioned above, the muon magnetic anomaly may also contain contributions from a new physics, which can be revealed, we hope, in a comparison of $a_{\mu}^{e x p}$ with an accurate theoretical evaluation of $a_{\mu}^{t h}$. While QED and weak interaction contributions to $a_{\mu}^{\text {th }}$ seem to be estimated very reliably, there is still opened a door for improvements in hadronic contributions.

As the most critical from all hadronic contributions are the light-by-light (LBL) meson pole terms [6], we recalculate the third-order hadronic LBL contributions to the anomalous magnetic moment of the muon $a_{\mu}^{\text {had }}$ from the pole terms of the scalar $\sigma, a_{0}$ and pseudoscalar $\pi^{0}, \eta, \eta^{\prime}$ mesons $(M)$ in the framework of the linearized extended Nambu-Jona-Lasinio model. The reason for the latter are predictions of series of recent papers

$$
\begin{aligned}
& a_{\mu}^{L B L}=(-52 \pm 18) \times 10^{-11} \quad[7] \\
& a_{\mu}^{L B L}=(-92 \pm 32) \times 10^{-11} \quad[8] \\
& a_{\mu}^{L B L}=(-79.2 \pm 15.4) \times 10^{-11} \quad[9] \\
& a_{\mu}^{L B L}=(+83 \pm 12) \times 10^{-11} \quad[10] \\
& a_{\mu}^{L B L}\left(\pi_{0}\right)=(+58 \pm 10) \times 10^{-11} \quad[10,11]
\end{aligned}
$$

which differ not only in the magnitude, but even in the sign. Moreover, in these papers only the pseudoscalar pole contributions were considered. In our paper we include the scalar meson $\left(\sigma, a_{0}\right)$ pole contributions as well.

The crucial point is description of transition form factor $\gamma^{*} \rightarrow M \gamma^{*}$. Current methods used for this aim are the ChPT and the vector-mesondominance (VMD) model.

In this paper the corresponding transition form factors by the constituent quark triangle loops with colourless and flavourless quarks with charge equal to the electron one are represented. (An application of a similar modified constituent quark triangle loop model for a prediction of the pion electromagnetic form factor behavior can be found in [12], where also a comparison with the naive VMD model prediction is carried out.) The mass of the quark in the triangle loop is always taken to be $m_{u}=m_{d}=m_{q}=(280 \pm 20) \mathrm{MeV}$ [13], which was determined in the framework of the chiral quark model of the Nambu-Jona-Lasinio type by exploiting the experimental values of the pion decay constant, the $\rho$-meson decay into two-pions constant, the masses 
of pion and kaon and the mass difference of $\eta$ and $\eta^{\prime}$ mesons. The unknown strong coupling constants of $\pi^{0}, \eta, \eta^{\prime}$ and $a_{0}$ mesons with quarks are evaluated in a comparison of the corresponding theoretical two-photon widths with experimental ones. The $\sigma$-meson coupling constant is taken to be equal to $\pi^{0}$-meson coupling constant as it follows from the corresponding Lagrangian. The $\sigma$-meson mass is taken to be $m_{\sigma}=(496 \pm 47) \mathrm{MeV}$ as an average of the values recently obtained experimentally from the decay $D^{+} \rightarrow \pi^{-} \pi^{+} \pi^{+}$14 and excited $\Upsilon$ decay 15 processes.

As a result we present explicit formulas for $a_{\mu}^{L B L}(M)\left(M=\pi^{0}, \eta, \eta^{\prime}, \sigma, a_{0}\right)$ in terms of Feynman parametric integrals of 10-dimensional order, which subsequently are calculated by MIKOR method [16].

The paper is organized as follows. In the next section all definitions and a derivation of basic relations is presented. More detail can be found in Appendix A and in Appendix B. The last section to numerical results and discussion is devoted.

\section{Meson pole terms in light-by-light contri- butions to $a_{\mu}^{\text {had }}$}

The third QED order hadronic LBL scattering contributions to $a_{\mu}^{\text {had }}$ are generally represented by the diagram in Fig. 1 $\mathcal{A}$, which contains a class of pseudoscalar mesons $\left(\pi^{ \pm}, K^{ \pm}\right.$and also $\left.K_{S, L}^{0}\right)$ square loop diagrams (Fig. 四B), a class of quark square loop diagrams (Fig. $\mathbb{1} \mathcal{C}$ ) and scalar $\sigma, a_{0}(980)$ and pseudoscalar $\pi^{0}, \eta, \eta^{\prime}$ meson pole diagrams (Fig. 2), where the off-shell structure of the photon-photon-meson vertices is taken into account by means of flavourless and colourless constituent quark triangle loops. Here the interaction of mesons (M) with quarks is described by the linearized Nambu-JonaLasinio type Lagrangian

$$
\mathcal{L}_{q \bar{q} M}=g_{M} \bar{q}(x)\left[\sigma(x)+i \pi(x) \gamma_{5}\right] q(x),
$$

with unknown strong coupling constant $g_{M}$. The latter for $\pi^{0}, \eta, \eta^{\prime}$ and $a_{0}$ mesons are evaluated by a comparison of the corresponding theoretical two-photon widths with the experimentally determined values [1].

For the on-mass shell scalar $(S)$ and pseudoscalar $(P)$ mesons decays one can write the following matrix elements

$$
\begin{aligned}
M\left(S(p) \rightarrow \gamma\left(k_{1}\right)+\gamma\left(k_{2}\right)\right)= & -\frac{i \alpha g_{S}}{\pi m_{q}} K\left(m_{S} / m_{q}\right)\left(k_{1} k_{2} g_{\mu \nu}-\right. \\
& \left.-k_{1 \nu} k_{2 \mu}\right) e_{1 \nu}\left(k_{1}\right) e_{2 \mu}\left(k_{2}\right),
\end{aligned}
$$



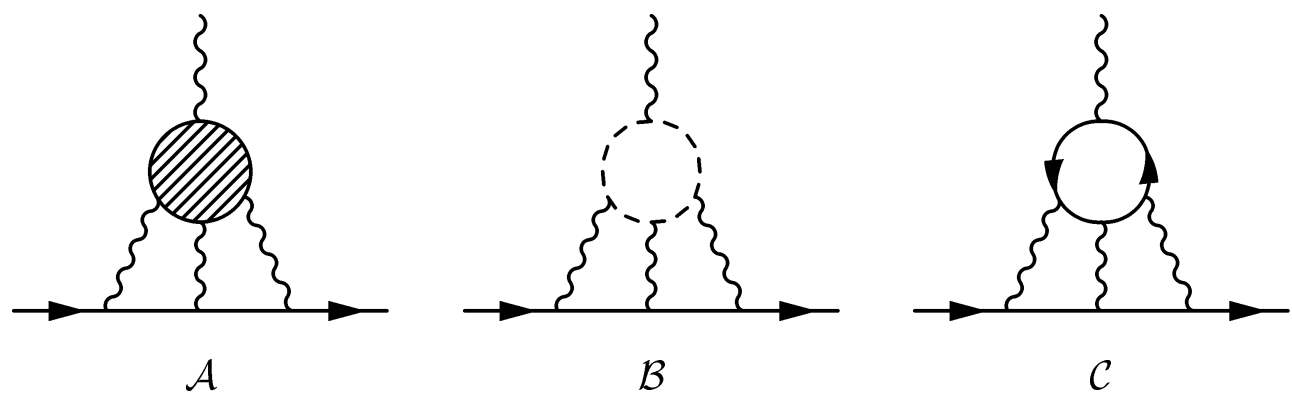

Figure 1: Third order hadronic light-by-light scattering contribution to $a_{\mu}^{\text {had }}$ $(\mathcal{A})$ and class of pseudoscalar meson square loop diagrams $(\mathcal{B})$ and quark square loop diagrams $(\mathcal{C})$ contributing to $(\mathcal{A})$.

$$
M\left(P(p) \rightarrow \gamma\left(k_{1}\right)+\gamma\left(k_{2}\right)\right)=\frac{\alpha g_{P}}{\pi m_{q}} J\left(m_{P} / m_{q}\right)\left(k_{1} k_{2} e_{1} e_{2}\right)
$$

with

$$
K(z)=2 \int_{0}^{1} d x \int_{0}^{1-x} \frac{1-4 x y}{1-x y z^{2}} d y, \quad J(z)=\frac{2}{z^{2}} \int_{0}^{1} \frac{d x}{x} \ln \left[1-z^{2} x(1-x)\right]
$$

and

$$
p^{2}=m_{M}^{2}, k_{1}^{2}=k_{2}^{2}=0,(a b c d)=\epsilon_{\alpha \beta \gamma \delta} a^{\alpha} b^{\beta} c^{\gamma} d^{\delta} .
$$

They lead to the theoretical two-photon widths (for more detail see Appendix A)

$$
\begin{aligned}
\Gamma_{S}^{\gamma \gamma} & =\frac{\alpha^{2} m_{S}^{3} g_{S}^{2}}{64 \pi^{3} m_{q}^{2}} K^{2}\left(m_{S} / m_{q}\right) \\
\Gamma_{P}^{\gamma \gamma} & =\frac{\alpha^{2} m_{P}^{3} g_{P}^{2}}{64 \pi^{3} m_{q}^{2}} J^{2}\left(m_{P} / m_{q}\right) .
\end{aligned}
$$

dependent on the scalar $g_{S}$ and pseudoscalar $g_{P}$ meson coupling constants with quarks, respectively.

Taking the meson masses with errors and the following world averaged values of the two-photon widths from the last Review of Particle Physics [1], $\Gamma_{\pi^{0}}^{\gamma \gamma}=(0.008 \pm 0) \mathrm{keV}, \Gamma_{\eta}^{\gamma \gamma}=(0.464 \pm 0.044) \mathrm{keV}, \Gamma_{\eta^{\prime}}^{\gamma \gamma}=(4.282 \pm 0.339) \mathrm{keV}$ and $\Gamma_{a_{0}}^{\gamma \gamma}=(0.24 \pm 0.08) \mathrm{keV}$, the quark mass value $m_{q}=(280 \pm 20) \mathrm{MeV}$, one finds the meson coupling constants with quarks as follows

$$
\begin{aligned}
& g_{\pi^{0}}^{2}=9.120 \pm 1.305, g_{\eta}^{2}=2.220 \pm 0.378 \\
& g_{\eta^{\prime}}^{2}=6.708 \pm 1.096, g_{a_{0}}^{2}=0.757 \pm 0.277
\end{aligned}
$$




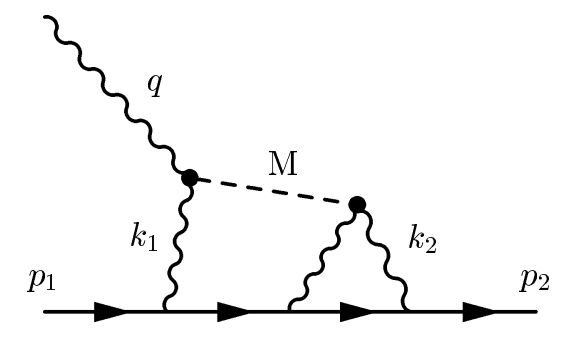

$\mathcal{A}$

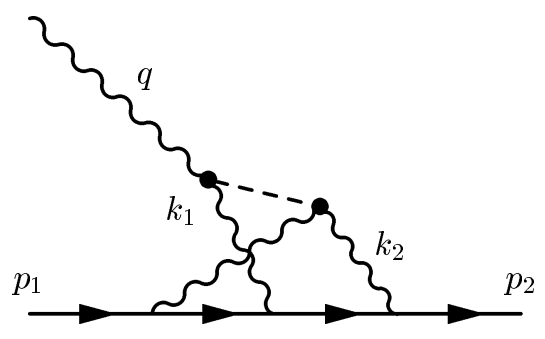

$\mathcal{C}$

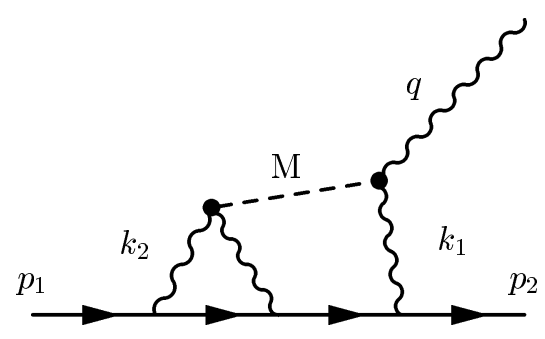

$\mathcal{B}$

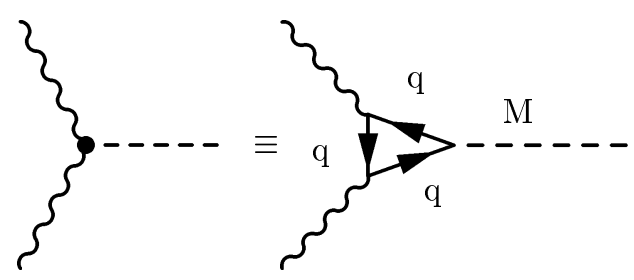

Figure 2: Meson (M) pole diagrams in the third order hadronic light-by-light scattering contributions to $a_{\mu}^{\text {had }}$.

Since there is no experimental result on the $\sigma$-meson decay into twophotons up to now, we identify $g_{\sigma}^{2}$ with $g_{\pi^{0}}^{2}$ as it follows from the Lagrangian (7).

Now we are ready to evaluate LBL meson pole terms contributions to $a_{\mu}^{\text {had }}$.

Usually the fermion-photon vertex function is written in the form

$$
\begin{gathered}
\Gamma_{\mu}=\bar{u}\left(p_{2}\right) \hat{V}_{\mu}(q) u\left(p_{1}\right), \\
\hat{V}_{\mu}(q)=\gamma_{\mu} F_{1}\left(q^{2}\right)+\frac{1}{4 m}\left[\hat{q}, \gamma_{\mu}\right] F_{2}\left(q^{2}\right), \quad q=p_{2}-p_{1},
\end{gathered}
$$

with on mass-shell muons, i.e. $p_{1}^{2}=p_{2}^{2}=m^{2}$ ( $m$ is the muon mass). Then the expression for the muon anomalous magnetic moment is

$$
\begin{aligned}
a_{\mu} & =\frac{(g-2)_{\mu}}{2}=F_{2}(0) \\
& =-\frac{3}{16 m^{2}} \lim _{q^{2} \rightarrow 0} \frac{1}{q^{2}} S p\left(\hat{p}_{2}+m\right) \hat{V}_{\mu}(q)\left(\hat{p}_{1}+m\right) \mathcal{P}_{\mu}(q),
\end{aligned}
$$

with a projection operator

$$
\mathcal{P}_{\mu}(q)=q^{2} \gamma_{\mu}+\frac{m}{3}\left[\hat{q}, \gamma_{\mu}\right]
$$


The gauge invariant set of Feynman diagrams containing the LBL scattering block with meson pole intermediate states is drawn in Fig. 2.

Their contributions to $a_{\mu}^{\text {had }}$ are

$$
\begin{aligned}
a_{\mu}^{L B L}(S)+a_{\mu}^{L B L}(P) & = \\
& -\frac{3}{16 m^{2}} \lim _{q^{2} \rightarrow 0} \frac{1}{q^{2}} S p\left(\hat{p}_{2}+m\right) \hat{V}_{\mu}^{S+P}\left(\hat{p}_{1}+m\right) \mathcal{P}_{\mu}(q),
\end{aligned}
$$

with

$$
\begin{array}{r}
\hat{V}_{\mu}^{S+P}=\frac{\alpha}{2^{6} \pi^{3}} \int \frac{d^{4} k_{1} d^{4} k_{2}}{\left(i \pi^{2}\right)^{2}} O^{\nu \lambda \sigma}\left[\frac{T_{\mu \nu \lambda \sigma}^{(P)}}{k_{1}^{2}-m_{P}^{2}}+\frac{T_{\mu \nu \lambda \sigma}^{(S)}}{k_{1}^{2}-m_{S}^{2}}\right] \frac{1}{k_{1}^{2} k_{2}^{2}\left(k_{1}-k_{2}\right)^{2}}, \\
O=2 O_{1}+O_{2}, \\
O_{1}^{\nu \lambda \sigma}=\gamma^{\sigma} \frac{\hat{p}_{2}-\hat{k}_{2}+m}{\left(p_{2}-k_{2}\right)^{2}-m^{2}} \gamma^{\lambda} \frac{\hat{p}_{1}-\hat{k}_{1}+m}{\left(p_{1}-k_{1}\right)^{2}-m^{2}} \gamma^{\nu}, \\
O_{2}^{\nu \lambda \sigma}=\gamma^{\sigma} \frac{\hat{p}_{2}-\hat{k}_{2}+m}{\left(p_{2}-k_{2}\right)^{2}-m^{2}} \gamma^{\nu} \frac{\hat{p}_{1}+\hat{k}_{1}-\hat{k}_{2}+m}{\left(p_{1}+k_{1}-k_{2}\right)^{2}-m^{2}} \gamma^{\lambda}
\end{array}
$$

where

$$
T_{\mu \nu \lambda \sigma}^{(P)}=\frac{4 \alpha^{2} g_{P}^{2}}{\pi^{2} m_{q}^{2}}\left(\mu \nu k_{1} q\right)\left(\lambda \sigma k_{2} k_{1}\right) f_{1}\left(k_{1}^{2}\right) f_{2}\left(k_{1}, k_{2}\right)
$$

with

$$
\begin{array}{r}
f_{1}\left(k_{1}^{2}\right)=\int_{0}^{1} \frac{m_{q}^{2}}{m_{q}^{2}-x(1-x) k_{1}^{2}} d x, f_{2}\left(k_{1}, k_{2}\right)=2 \int_{0}^{1} d x_{1} \int_{0}^{1-x_{1}} \frac{m_{q}^{2}}{d} d x_{2}, \\
d=m_{q}^{2}-k_{2}^{2} x_{3}\left(1-x_{3}\right)-k_{1}^{2} x_{2}\left(1-x_{2}\right)+2 k_{1} k_{2} x_{2} x_{3}, \\
x_{3}=1-x_{1}-x_{2} .
\end{array}
$$

Here we would like to note that the functions $f_{1}\left(k_{1}^{2}\right), f_{2}\left(k_{1}, k_{2}\right)$ at the limit of an infinite value of the quark mass, $m_{q} \rightarrow \infty$, tend to one.

The expression for $T^{(S)}$ has a similar form

$$
\begin{gathered}
T_{\mu \nu \lambda \sigma}^{(S)}=-\frac{4 \alpha^{2} g_{S}^{2}}{\pi^{2} m_{q}^{2}}\left(g_{\mu \nu} k_{1} q-k_{1 \mu} q_{\nu}\right) \tilde{f}_{1}\left(k_{1}^{2}\right) f_{\lambda \sigma}\left(k_{1}, k_{2}\right), \\
\tilde{f}_{1}\left(k_{1}^{2}\right)=\int_{0}^{1} \frac{m_{q}^{2}(1-2 x(1-x)) d x}{m_{q}^{2}-x(1-x) k_{1}^{2}}, \\
f_{\lambda \sigma}\left(k_{1}, k_{2}\right)=2 \int_{0}^{1} d x_{1} \int_{0}^{1-x_{1}} \frac{m_{q}^{2}}{d}\left\{( 1 - 4 x _ { 1 } x _ { 2 } ) \left[g_{\lambda \sigma} k_{2}\left(k_{1}-k_{2}\right)-\right.\right. \\
\left.\left.k_{2 \lambda}\left(k_{1}-k_{2}\right)_{\sigma}\right]+2\left(k_{1}-k_{2}\right)^{2} x_{1}\left(2 x_{1}-1\right) g_{\lambda \sigma}\right\} d x_{2} .
\end{gathered}
$$


Usual procedure of performing the loop-momenta integration using the Feynman parameters method (see Appendix B) leads to

$$
a_{\mu}^{L B L}(M)=-R_{M} F_{M}\left(m_{M}, m_{q}\right)
$$

where

$$
R_{M}=\frac{3 m^{2} \alpha^{2} g_{M}^{2}}{64 \pi^{5} m_{q}^{2}}
$$

and

$$
F_{P}\left(m_{P}, m_{q}\right)=2 M_{a}+M_{b}, \quad F_{S}\left(m_{S}, m_{q}\right)=2 N_{a}+N_{b} .
$$

The $M_{i}, N_{i}$ are given by the expressions

$$
\begin{gathered}
M_{a}=\int d \Gamma_{a} \frac{1}{z D_{1}^{2}}\left[\frac{A_{a}^{P}}{D_{1}}+B_{a}^{P}\right] \\
M_{b}=\int d \Gamma_{b} \frac{1}{\sigma^{2} D_{2}^{2}}\left[\frac{A_{b}^{P}}{D_{2}}+B_{b}^{P}\right] \\
N_{a}=\int d \Gamma_{a} \frac{1}{z D_{1}^{2}}\left[\frac{A_{a}^{S}}{D_{1}}+B_{a}^{S}\right] \\
N_{b}=\int d \Gamma_{b} \frac{1}{\sigma^{2} D_{2}^{2}}\left[\frac{A_{b}^{S}}{D_{2}}+B_{b}^{S}\right],
\end{gathered}
$$

with $d \Gamma_{a, b}$ the 10-dimensional integrals on Feynman parameters

$$
\begin{gathered}
\int d \Gamma_{a}=\tau^{4} \int_{0}^{1} \frac{d x}{x \bar{x}} \int_{0}^{1} d x_{1} \int_{0}^{\bar{x}_{1}} \frac{d x_{3}}{x_{3} \bar{x}_{3}} \int_{0}^{1} d z_{1} \int_{0}^{\bar{z}_{1}} d z_{2} \int_{0}^{1} \alpha_{2} \alpha_{3}^{2} d^{3} \alpha d y \rho \bar{\rho}^{2} d \rho \\
\int d \Gamma_{b}=\tau^{4} \int_{0}^{1} \frac{d x}{x \bar{x}} \int_{0}^{1} d x_{1} \int_{0}^{\bar{x}_{1}} \frac{d x_{3}}{x_{3} \bar{x}_{3}} \int_{0}^{1} d z_{1} \int_{0}^{\bar{z}_{1}} d z_{2} \int_{0}^{1} \alpha_{2} \alpha_{3}^{2} \alpha_{4}^{3} d^{4} \alpha \rho \bar{\rho}^{2} d \rho, \\
\bar{x}=1-x, \bar{z}_{1}=1-z_{1}, \tau=\frac{m_{q}}{m}, d^{3} \alpha=d \alpha_{1} d \alpha_{2} d \alpha_{3}, d^{4} \alpha=d \alpha_{1} d \alpha_{2} d \alpha_{3} d \alpha_{4} .
\end{gathered}
$$

The explicit expressions for polynomials $A, B$ are

$$
\begin{aligned}
A_{a}^{P} & =\frac{8 y \rho}{3 z}\left[2 a_{1}^{2}+2 r_{1} a_{1}\left(a_{1}+b_{1}\right)-r_{1}^{2} a_{1} b_{1}\right]-8 r_{1}^{3}, \\
B_{a}^{P} & =4+12 r_{1}+4 a_{1} b_{1} \frac{y \rho}{z}, \\
A_{b}^{P} & =\frac{8 \rho}{3 \sigma}\left[a_{2}^{3}-r_{2}^{2} a_{2} b_{2}\left(b_{2}-1\right)\right]-\frac{16}{3} r_{2}^{2} a_{2}+\frac{8}{3} r_{2}^{3}\left(1-2 b_{2}\right), \\
B_{a}^{P} & =4 a_{2}-\frac{8}{3}+r_{2}\left(8 b_{2}-4\right)+\frac{4 \rho}{\sigma} a_{2} b_{2}\left(b_{2}-1\right),
\end{aligned}
$$




$$
\begin{aligned}
A_{a}^{S} & =\frac{y \rho}{z}\left[-8 a_{1}^{3}-16 r_{1} a_{1}\left(a_{1} b_{1}-\frac{1}{3} a_{1}+\frac{1}{3} b_{1}\right)\right. \\
& \left.-8 r_{1}^{2}\left(a_{1} b_{1}-\frac{2}{3} a_{1}+\frac{2}{3} b_{1}-\frac{2}{3}\right) b_{1}\right] d_{1}\left(x_{1}, x_{2}\right) \\
& +\frac{y \rho}{z}\left[(16 / 3) a_{1}^{2}\left(a_{1}+1\right)+\frac{32}{3} r_{1}\left(b_{1}-1\right)\left(a_{1}+1\right) a_{1}\right. \\
& \left.+r_{1}^{2} \frac{16}{3}\left(a_{1}+1\right)\left(b_{1}-1\right)^{2}\right] d_{2}\left(x_{1}\right) \\
B_{a}^{S} & =\left(8 a_{1}+\frac{16}{3}\right) d_{2}\left(x_{1}\right)-\left(4+12 a_{1}\right) d_{1}\left(x_{1}, x_{2}\right) \\
& -\frac{8 y \rho}{z}\left(a_{1}+1\right)\left(b_{1}-1\right)^{2} d_{2}\left(x_{1}\right) \\
& +\frac{y \rho}{z}\left(12 a_{1} b_{1}-8 a_{1}+8 b_{1}-8\right) b_{1} d_{1}\left(x_{1}, x_{2}\right) \\
A_{b}^{S} & =\frac{\rho}{\sigma}\left\{\left[\frac{16}{3} a_{2}^{2}\left(a_{2}-1\right)+\frac{32}{3} r_{2} a_{2}\left(a_{2}-1\right)\left(b_{2}-1\right)\right.\right. \\
& \left.+\frac{16}{3} r_{2}\left(a_{2}-1\right)\left(b_{2}-1\right)^{2}\right] d_{2}\left(x_{1}\right) \\
& \left.+\left[-\frac{16}{3} a_{2}^{2}+\frac{16}{3} r_{2}^{2} b_{2}\left(b_{2}-1\right)\right] d_{1}\left(x_{1}, x_{2}\right)\right\} \\
B_{b}^{S} & =\left[\frac{8}{3}-\frac{8 \rho}{\sigma} b_{2}\left(b_{2}-1\right)\right] d_{1}\left(x_{1}, x_{2}\right) \\
& +\left[8 a_{2}-\frac{16}{3}-\frac{8 \rho}{\sigma}\left(a_{2}-1\right)\left(b_{2}-1\right)^{2}\right] d_{2}\left(x_{1}\right) \\
& {\left[\frac{8}{3}\right) }
\end{aligned}
$$

with

$$
\begin{aligned}
& a_{1}=\alpha_{3} \bar{\alpha}_{2}, b_{1}=\alpha_{2} \alpha_{3} \gamma_{1}, a_{2}=\alpha_{4}\left(\alpha_{3} \bar{\alpha}_{2}+\bar{\alpha}_{3}\right), \\
& b_{2}=\alpha_{4}\left(\alpha_{3} \gamma_{1}+\bar{\alpha}_{3}\right), r_{1}=\rho\left(\bar{y}-c_{1} y\right), r_{2}=-c_{2} \rho, \\
& d_{1}\left(x_{1}, x_{2}\right)=1-4 x_{1} x_{2}, d_{2}\left(x_{1}\right)=2 x_{1}\left(2 x_{1}-1\right) .
\end{aligned}
$$

Quantities $c_{1,2}, \gamma_{1}, z, D_{1}, D_{2}$ and $\sigma$ are given in Appendix B. 


\section{Results and discussion}

The result for the meson pole contributions of LBL type has the form

$$
a_{\mu}^{L B L}(M)=a_{\mu}^{L B L}\left(\pi^{0}\right)+a_{\mu}^{L B L}(\eta)+a_{\mu}^{L B L}\left(\eta^{\prime}\right)+a_{\mu}^{L B L}(\sigma)+a_{\mu}^{L B L}\left(a_{0}\right)
$$

where

$$
\begin{aligned}
a_{\mu}^{L B L}\left(\pi^{0}\right) & =-R_{\pi^{0}} F_{\pi^{0}}\left(m_{\pi^{0}}, m_{q}\right) \\
a_{\mu}^{L B L}(\eta) & =-R_{\eta} F_{\eta}\left(m_{\eta}, m_{q}\right) \\
a_{\mu}^{L B L}\left(\eta^{\prime}\right) & =-R_{\eta^{\prime}} F_{\eta^{\prime}}\left(m_{\eta^{\prime}}, m_{q}\right) \\
a_{\mu}^{L B L}(\sigma) & =-R_{\sigma} F_{\sigma}\left(m_{\sigma}, m_{q}\right) \\
a_{\mu}^{L B L}\left(a_{0}\right) & =-R_{a_{0}} F_{a_{0}}\left(m_{a_{0}}, m_{q}\right) .
\end{aligned}
$$

Now, taking the values (13) of $g_{M}^{2}$ determined in Section 2, the mass of the quark $m_{q}=(280 \pm 20) \mathrm{MeV}$ [13], the mass of the $\sigma$-meson $m_{\sigma}=$ $(496 \pm 47) \mathrm{MeV}$ [14, 15], the mass of the muon and the corresponding scalar and pseudoscalar mesons from the Review of Particle Physics [1] one finds

$$
\begin{aligned}
a_{\mu}^{L B L}\left(\pi^{0}\right) & =(81.83 \pm 16.50) \times 10^{-11} \\
a_{\mu}^{L B L}(\eta) & =(5.62 \pm 1.25) \times 10^{-11} \\
a_{\mu}^{L B L}\left(\eta^{\prime}\right) & =(8.00 \pm 1.74) \times 10^{-11} \\
a_{\mu}^{L B L}(\sigma) & =(11.67 \pm 2.38) \times 10^{-11} \\
a_{\mu}^{L B L}\left(a_{0}\right) & =(0.62 \pm 0.24) \times 10^{-11} .
\end{aligned}
$$

Here we would like to note that our value for the $\pi^{0}$ is in the framework of error bars consistent with [10, 11.

So, the total contribution of meson poles in LBL is

$$
a_{\mu}^{L B L}(M)=(107.74 \pm 16.81) \times 10^{-11},
$$

where the resultant error is the addition in quadrature of all partial errors of (30).

Together with the contributions of the pseudoscalar meson $\left(\pi^{ \pm}, K^{ \pm}, K_{S, L}^{0}\right)$ square loops and constituent quark square loops [7, 8] it gives

$$
a_{\mu}^{L B L}(\text { total })=(111.20 \pm 16.81) \times 10^{-11} .
$$

The others 3-loop hadronic contributions derived from the hadronic vacuum polarizations $(V P)$ were most recently evaluated by Krause 17

$$
a_{\mu}^{(3) V P}=(-101 \pm 6) \times 10^{-11} .
$$


Then the total 3-loop hadronic correction from (32) and (33) is

$$
a_{\mu}^{(3) h a d}=a_{\mu}^{L B L}(\text { total })+a_{\mu}^{(3) V P}=(10.20 \pm 17.28) \times 10^{-11}
$$

where the errors have been again added in quadratures.

If we take into account the most recent evaluation [18] of the lowestorder hadronic vacuum-polarization contribution to the anomalous magnetic moment of the muon

$$
a_{\mu}^{(2) h a d}=(7021 \pm 76) \times 10^{-11}
$$

(it is interesting to notice that this result almost coincides in the central value and in the error as well with the result [19 of one of the authors (S.D.) of this paper obtained 12 years ago) the pure QED contribution up to 8th order [20]

$$
a_{\mu}^{Q E D}=(116584705.7 \pm 2.9) \times 10^{-11}
$$

and the single- and two-loop weak interaction contribution [5], finally one gets the SM theoretical prediction of the muon anomalous magnetic moment value to be

$$
a_{\mu}^{\text {th }}=(116591888.9 \pm 78.1) \times 10^{-11} .
$$

Comparing this theoretical result with experimental one (3) one finds

$$
a_{\mu}^{e x p}-a_{\mu}^{t h}=(131 \pm 178) \times 10^{-11}
$$

which implies very good consistency of the SM prediction for the anomalous magnetic moment of the muon with experiment.

\section{Appendix A}

We put here the details of calculations of the two-photon widths in the framework of the linearized $\mathrm{Nambu}-$ Jona-Lasinio model.

The decay matrix element of a pseudoscalar meson $P$ into two photons has a form (we take into account two directions of fermion line in the triangle fermion loop)

$$
M\left(P(p) \rightarrow \gamma\left(e_{1 \lambda}\left(k_{1}\right)\right) \gamma\left(e_{2 \sigma}\left(k_{2}\right)\right)=\frac{2 i \alpha g_{P} m_{q}}{\pi} \int \frac{d^{4} k}{i \pi^{2}} \frac{S_{1}}{a_{1} a_{2} a_{3}},\right.
$$

with

$$
\begin{array}{r}
S_{1}=\frac{1}{4 m_{q}} \operatorname{Tr}\left(\hat{k}-\hat{k}_{2}+m_{q}\right) \hat{e}_{2}\left(\hat{k}+m_{q}\right) \hat{e}_{1}\left(\hat{k}+\hat{k}_{1}+m_{q}\right) \gamma_{5}=i\left(k_{2} k_{1} e_{1} e_{2}\right) \\
a_{1}=\left(k+k_{1}\right)^{2}-m_{q}^{2}, a_{2}=\left(k_{1}-k_{2}\right)^{2}-m_{q}^{2}, a_{3}=k^{2}-m_{q}^{2}
\end{array}
$$


where we use the definitions $\gamma_{5}=i \gamma^{0} \gamma^{1} \gamma^{2} \gamma^{3}, \frac{1}{4} S p \gamma_{\mu} \gamma_{\nu} \gamma_{\lambda} \gamma_{\sigma}=i \varepsilon_{\mu \nu \lambda \sigma}, \varepsilon_{0123}=$ +1 .

Joining the denominators and performing the loop momentum integration one obtains

$$
M_{P}^{\gamma^{*} \gamma^{*}}=-\frac{2 g_{P} \alpha}{\pi m_{q}} \int \frac{d^{3} x \delta\left(\sum x-1\right) m_{q}^{2}\left(k_{2} k_{1} e_{1} e_{2}\right)}{m_{q}^{2}-x_{1} x_{2} p^{2}-x_{1} x_{3} k_{2}^{2}-x_{2} x_{3} k_{1}^{2}} .
$$

This expression is used for the construction of $f_{2}\left(k_{1}, k_{2}\right)$ in (19).

For the case of real particles we have

$$
M_{P}^{\gamma \gamma}=\frac{\alpha g_{P}}{\pi m_{q}}\left(k_{1} k_{2} e_{1} e_{2}\right) J\left(m_{P} / m_{q}\right)
$$

with $J(z)$ given by $(10)$.

Note that for the case of $\eta^{\prime}$-meson the matrix element becomes complex. Numerically one obtains

$$
J^{2}\left(m_{\pi^{0}} / m_{q}\right)=1.04, J^{2}\left(m_{\eta} / m_{q}\right)=3.73,\left|J\left(m_{\eta^{\prime}} / m_{q}\right)\right|^{2}=2.12 .
$$

For the matrix element of a decay of the scalar meson $S$ into two photons one can write

$$
M\left(S(p) \rightarrow \gamma\left(k_{1}, e_{1}\right) \gamma\left(k_{2}, e_{2}\right)\right)=e_{1 \mu} e_{2 \nu} M^{\mu \nu}
$$

where

$$
\begin{gathered}
M^{\mu \nu}=\frac{2 i \alpha g_{S} m_{q}}{\pi} \int \frac{d^{4} k}{i \pi^{2}} \frac{T^{\mu \nu}}{a_{1} a_{2} a_{3}} \\
T^{\mu \nu}=\frac{1}{4 m_{q}} S p\left(\hat{k}-\hat{k}_{2}+m_{q}\right) \gamma_{\nu}\left(\hat{k}+m_{q}\right) \gamma_{\mu}\left(\hat{k}+\hat{k}_{2}+m_{q}\right) .
\end{gathered}
$$

This quantity suffers from the ultraviolet divergences. It is the gauge invariance requirement which provides the regularization. General form of $M^{\mu \nu}\left(k_{1}, k_{2}\right)$, which satisfies the current conservation condition

$$
M^{\mu \nu}\left(k_{1}, k_{2}\right)\left[k_{1 \mu} ; k_{2 \nu}\right]=0,
$$

has the form

$$
\begin{aligned}
M^{\mu \nu}\left(k_{1}, k_{2}\right)= & A\left(g^{\mu \nu} k_{1} k_{2}-k_{1}^{\nu} k_{2}^{\mu}\right) \\
& +B\left(k_{1}^{2} k_{2}^{\nu}-k_{1} k_{2} k_{1}^{\nu}\right)\left(k_{2}^{2} k_{1}^{\mu}-k_{1} k_{2} k_{2}^{\mu}\right) .
\end{aligned}
$$

Usual procedure of joining the denominators, the calculation of the trace, a performation of the loop momentum integration and an application of the gauge conditions to the muon block, leads to a final result. 
For the case of real particles the structure $B$ disappears and we have

$$
M^{(S \rightarrow \gamma \gamma)}=e_{1}^{\mu}\left(k_{1}\right) e_{2}^{\nu}\left(k_{2}\right)\left(g_{\mu \nu} k_{1} k_{2}-k_{1}^{\nu} k_{2}^{\mu}\right) \frac{-i \alpha g_{S}}{\pi m_{q}} K\left(m_{S} / m_{q}\right),
$$

which leads to the expression (11) for the width with $K(z)$ given by (10). Numerically

$$
\left|K\left(m_{a^{0}} / m_{q}\right)\right|^{2}=0.96
$$

\section{Appendix B}

For a performance of the loop momenta integration we use Feynman trick of "joining" the denominators

$$
\frac{1}{a^{m} b^{n}}=\frac{(n+m-1) !}{(n-1) !(m-1) !} \int_{0}^{1} \frac{x^{m-1}(1-x)^{n-1} d x}{(a x+b(1-x))^{m+n}}
$$

and the relation

$$
\int \frac{d^{4} k}{i \pi^{2}} \frac{\left(k^{2}\right)^{m}}{\left(k^{2}-d\right)^{n}}=(-1)^{m+n} \frac{(m+1) !(n-m-3) !}{(n-1) ! d^{n-m-2}}, n>m+2 .
$$

For this aim we present the denominators containing $k_{2}$ in the form

$$
\frac{1}{-x_{3} \bar{x}_{3}} \frac{1}{\{1\}\{2\}\{3\}\{4\}}\left[\frac{1}{\{5\}} ; \frac{1}{\{6\}}\right]
$$

where

$$
\begin{array}{r}
\{1\}=k_{2}^{2},\{2\}=k_{2}^{2}-2 p_{2} k_{2},\{3\}=\left(k_{2}-k_{1}\right)^{2},\{4\}=d /\left(-x_{3} \bar{x}_{3}\right) \\
\{5\}=k_{1}^{2}-2 p_{1} k_{1} ;\{6\}=\left(p_{1}+k_{1}-k_{2}\right)^{2}-m^{2}, \bar{x}=1-x .
\end{array}
$$

Using Feynman parameter $\alpha_{1}$ to join the denominators $\{3\},\{4\}$ and the parameter $\alpha_{2}$ for joining the result with $\{2\}$ we have

$$
\begin{array}{r}
\alpha_{2}\left[\alpha_{1}\{4\}+\bar{\alpha}_{1}\{3\}\right]+\bar{\alpha}_{2}\{2\}=k_{2}^{2}-2 k_{2} a+\alpha_{2} \Delta_{1}, a=\alpha_{2} \gamma_{1} k_{1}+\bar{\alpha}_{2} p_{2}, \\
\Delta_{1}=\alpha_{2} \alpha_{1} \frac{x_{2} \bar{x}_{2} k_{1}^{2}-m_{q}^{2}}{x_{3} \bar{x}_{3}}+\alpha_{2} \bar{\alpha}_{2} k_{1}^{2}, \gamma_{1}=\bar{\alpha}_{1}+\alpha_{1} \frac{x_{2}}{\bar{x}_{3}}, \bar{a}=1=a .
\end{array}
$$

For the diagram in Fig. $2 \mathcal{A}$ we use the parameter $\alpha_{3}$ for the joining of the denominator $\{1\}$ with the above result

$$
\begin{gathered}
\left(k_{2}-b\right)^{2}-z d_{1}, \\
b=\alpha_{2} a, z=\left(\alpha_{2} \alpha_{3} \gamma_{1}\right)^{2}-\alpha_{2} \alpha_{3}\left(\bar{\alpha}_{1}+\frac{\alpha_{1} x_{2} \bar{x}_{2}}{x_{3} \bar{x}_{3}}\right), \\
d_{1}=k_{1}^{2}+2 c_{1} k_{1} p_{1}+\Delta_{2}, \\
c_{1}=\frac{\alpha_{2} \alpha_{3}^{2} \bar{\alpha}_{2} \gamma_{1}}{z}, \Delta_{2}=\frac{1}{z}\left[m^{2} \alpha_{3}^{2} \bar{\alpha}_{2}^{2}+m_{q}^{2} \frac{\alpha_{1} \alpha_{2} \alpha_{3}}{x_{3} \bar{x}_{3}}\right] .
\end{gathered}
$$


For the diagram in Fig. $2 \mathcal{B}$ we use the parameter $\alpha_{3}$ for the joining of the denominator (B.3) with $\{6\}$ and parameter $\alpha_{4}$ to join $\{1\}$ with the result

$$
\left(k_{2}-b_{2}\right)^{2}-\sigma d_{2}
$$

where

$$
\begin{aligned}
\sigma & =\alpha_{4}^{2}\left(\alpha_{3} \gamma_{1}+\bar{\alpha}_{3}\right)^{2}-\alpha_{4} \bar{\alpha}_{3}-\alpha_{2} \alpha_{3} \alpha_{4}\left(\bar{\alpha}_{1}+\alpha_{1} \frac{x_{2} \bar{x}_{2}}{x_{3} \bar{x}_{3}}\right), \\
b_{2} & =\alpha_{4}\left[\left(\bar{\alpha}_{3}+\alpha_{1} \gamma_{1}\right) k_{1}+\left(\bar{\alpha}_{3}+\alpha_{3} \bar{\alpha}_{2}\right) p_{1}\right], \\
d_{2} & =k_{1}^{2}+2 c_{2} p_{1} k_{1}+\delta \\
c_{2} & =\frac{1}{\sigma} \alpha_{4}\left[\alpha_{4}\left(\alpha_{3} \gamma_{1}+\bar{\alpha}_{3}\right)\left(\alpha_{3} \bar{\alpha}_{2}+\bar{\alpha}_{3}\right)-\bar{\alpha}_{3}\right], \\
\delta & =\frac{1}{\sigma}\left[m^{2} \alpha_{4}^{2}\left(\alpha_{3} \bar{\alpha}_{2}+\bar{\alpha}_{3}\right)^{2}+\frac{\alpha_{1} \alpha_{2} \alpha_{3} \alpha_{4}}{x_{3} \bar{x}_{3}} m_{q}^{2}\right] .
\end{aligned}
$$

To perform the $k_{1}$ integration we use the parameters $z_{1}, z_{2}$ to join the denominators containing $k_{1}^{2}$

$$
\frac{1}{x \bar{x}} \frac{1}{k_{1}^{2}\left(k_{1}^{2}-M^{2}\right)\left(k_{1}^{2}-M_{1}^{2}\right)}=\frac{2}{x \bar{x}} \int_{0}^{1} d z_{1} \int_{0}^{\bar{z}_{1}} \frac{d z_{2}}{d_{3}^{3}},
$$

with

$$
d_{3}=k_{1}^{2}-z_{1} M_{1}^{2}-z_{2} M^{2}, M_{1}^{2}=\frac{m_{q}^{2}}{x \bar{x}}
$$

For the diagram in Fig. $2 \mathcal{B}$ we use parameter $\rho$ to join the denominators $d_{2}, d_{3}$

$$
\begin{gathered}
\rho d_{2}+\bar{\rho} d_{3}=\left(k_{1}-q_{2}\right)^{2}-m^{2} D_{2} ; \\
q_{2}=-\rho c_{2} p_{1}, m^{2} D_{2}=m^{2}\left(c_{2} \rho\right)^{2}-\delta \rho+\bar{\rho}\left(z_{1} M_{1}^{2}+z_{2} M^{2}\right) .
\end{gathered}
$$

For the diagram in Fig. $2 \mathcal{A}$ we use parameter $y$ to join $d_{1}$ with $\{5\}$

$$
y d_{1}+\bar{y}(5)=k_{1}^{2}-2 p_{1} k_{1}\left(\bar{y}-c_{1} y\right)+y \Delta_{1}
$$

and parameter $\rho$ to join the result with $d_{3}$. As a result one gets

$$
\begin{aligned}
& \left(k_{1}-q_{1}\right)^{2}-D_{1} m^{2} ; q_{1}=\rho\left(\bar{y}-c_{1} y\right) p_{1}, \\
& m^{2} D_{1}=m^{2} \rho^{2}\left(\bar{y}-y c_{1}\right)^{2}+\bar{\rho}\left(z_{1} M_{1}^{2}+z_{2} M^{2}\right)-\rho y \Delta_{1} .
\end{aligned}
$$

\section{Acknowledgments}

We are grateful to participants of BLTP Seminar for discussions. E. A. K is grateful to RFBR 01-02-17437 as well as NNC, JINR for financial support via INTAS Grant No. 97-30494 and to SR grant 2000.

The work was in part also supported by Slovak Grant Agency for Sciences, Grant No. 2/5085/20 (S. D.) and Grant No. 1/7068/20 (A. Z. D). 


\section{References}

[1] Review of Particle Phys., D. E. Groom at al., European Physical Journal C15 (2000) 1.

[2] Muon (g-2) Collaboration, H. N. Brown et al., Phys. Rev. Lett. 86 (2001) 2227.

[3] Muon (g-2) Collaboration, R. M. Carey et al., Phys. Rev. Lett. 82 (1999) 1632.

[4] A. Czarnecki, B. Krause and W. J. Marciano, Phys. Rev. Lett. 76 (1996) 3267.

[5] G. Degrassi and G. F. Giudice, Phys. Rev. D58 (1998) 53007.

[6] K. Melnikov, hep-ph/0105267

[7] M. Hayakawa, T. Kinoshita and A. I. Sanda, Phys. Rev. D54 (1996) 3137.

[8] J. Bijnens, E. Pallante and J. Prades, Nucl. Phys. B474 (1996) 379.

[9] M. Hayakawa and T. Kinoshita, Phys. Rev. D57 (1998) 465.

[10] M. Knecht and A. Nyffeler, hep-ph/0111058.

[11] I. Blokland, A. Czarnecki and K. Melnikov, hep-ph/0112117.

[12] S. Dubnička, G. Georgios and V. A. Meshcheryakov, Can. J. Phys. 63 (1985) 1357.

[13] M. Nagy, M. K. Volkov and V. L. Yudichev, Proc. of Int. Conf. "Hadron Structure 2000", Stara Lesna, Slovak Republic, 2.-7.10. 2000, Eds: A.-Z. Dubničková, S. Dubnička and P. Stríženec, Comenius Univ., Bratislava (2001) p. 188.

[14] E. M. Aitala et al., Phys. Rev. Lett. 86 (2001) 770.

[15] T. Komada, M. Ishida and S. Ishida, Phys. Lett. 508 B (2001) 31.

[16] I. M. Korobov: Number theory method in approximate analysis, FIZMATGIZ, Moscow (1963) (in Russian).

[17] B. Krause, Phys. Lett. 390 B (1997) 392.

[18] S. Narison, Phys. Lett. 513 B (2001) 53. 
[19] L. Martinovič and S. Dubnička, Phys. Rev. D 42 (1990) 884.

[20] V. W. Hughes and T. Kinoshita, Rev. Mod. Phys. 71 (2) (1999) S133. 